\title{
Uniportal thoracoscopic treatment in bronchiectasis patients: preliminary experience
}

\author{
Ilhan Ocakcioglu \\ Department of Thoracic Surgery, Marmara University Pendik Training and Research Hospital, Istanbul, Turkey
}

Videosurgery Miniinv 2019; 14 (2): 304-310

DOI: https://doi.org/10.5114/wiitm.2018.78971

\begin{abstract}
Introduction: Bronchiectasis is defined as a permanent and abnormal dilation of the bronchi due to destruction of the bronchial wall. The thoracoscopic approach is represented in the literature by a few reports, and multiple port incisions are observed in these studies.

Aim: To investigate the effectiveness and outcomes of the single port video-assisted thoracoscopic surgery (VATS) method in the surgical treatment of bronchiectasis. To our knowledge, this is the first study reporting surgical treatment of bronchiectasis via the uniportal thoracoscopic approach.

Material and methods: The medical records of 15 patients undergoing surgery for bronchiectasis between 2013 and 2017 were reviewed. The patients were evaluated with regard to age, gender, duration of symptoms, localization, types and duration of the surgery, presence of pleural adhesion, intraoperative complications, amount of intraoperative bleeding, conversion to open surgery, postoperative drainage amount, chest tube removal time, length of hospital stay, mortality and morbidity, follow-up period, duration of narcotic analgesic usage, pain according to visual analog scale evaluation and satisfaction score.

Results: A total of 15 patients initially underwent single port thoracoscopic resection and 14 procedures were completed thoracoscopically. In terms of anatomic resections, 6 patients underwent left lower lobectomy, 4 right lower lobectomy, 1 right upper lobectomy, 1 left lower lobectomy + lingulectomy, 1 right basal segmentectomy and 1 patient underwent lingulectomy. The mean operative time was $137.1 \pm 24.5$ min and the mean length of hospital stay was $4.78 \pm 1.52$ days. The mean postoperative analgesic requirement was $2.85 \pm 0.66$ days.

Conclusions: We believe that uniportal VATS is a safe, feasible, and effective technique for selected bronchiectasis patients.
\end{abstract}

Key words: video-assisted thoracoscopic surgery, minimally invasive surgery, bronchiectasis, uniportal.

\section{Introduction}

Bronchiectasis is defined as a permanent and abnormal dilation of the bronchi due to destruction of the elastic and muscular components of the bronchial wall [1]. It arises from recurrent transmural inflammation and infection in the bronchi. It is still a great problem in under-developed countries, although its incidence is $0.06 \%$ in developed countries [2]. Ear- ly stage and localized disease may be controlled through conservative approaches but some patients still need surgery.

Because of the presence of dense pleural adhesions and multiple lymph nodes, the open surgical approach is widely preferred in the surgical treatment of bronchiectasis [3]. The feasibility and safety of pulmonary resection via video-assisted thoracoscopic surgery (VATS) have been established for

\section{Address for correspondence}

Ilhan Ocakcioglu MD, Department of Thoracic Surgery, Marmara University Pendik Training and Research Hospital, 34700 Istanbul,

Turkey, phone: +90 505779 8691, e-mail: ilhanocakcioglu@hotmail.com 
lung cancer [4]. Unfortunately, there have been few reports regarding the use of VATS in the treatment of benign pulmonary diseases such as bronchiectasis. Moreover, multiple port incisions are observed in these studies $[3,5,6]$.

\section{Aim}

In this study, we aimed to investigate the effectiveness and outcomes of the single port VATS method in the surgical treatment of bronchiectasis. To our knowledge, this is the first study reporting surgical treatment of bronchiectasis via the uniportal thoracoscopic approach.

\section{Material and methods}

This study was a retrospective review of a prospectively maintained database. The medical records of 15 patients undergoing surgery for bronchiectasis between 2013 and 2017 were reviewed.

High resolution computed tomography (HRCT) was used for making the diagnosis of bronchiectasis (Photo 1). Furthermore, the patients were evaluated for adhesion and scarring with HRCT and their availability for the operation was determined.

The indications for surgical resection included the presence of localized bronchiectasis diagnosed through HRCT and a history of experiencing pulmonary infection requiring antibiotic use at least five times a year. A chronic cough, sputum production irresponsive to medical treatment, and hemoptysis were the other indications for surgery. Patients who had massive hemoptysis were not included in the study. The presence of bilateral diffuse lesions on HRCT or inadequate cardiopulmonary reserve was accepted as a contraindication.

The patients were evaluated according to the age, gender, duration of symptoms, localization, types and duration of the surgery, presence of pleural adhesion, intraoperative complications, amount of intraoperative bleeding, conversion to open surgery, postoperative drainage amount, chest tube removal time, length of hospital stay, mortality and morbidity, follow-up period, duration of narcotic analgesic (NA) usage, pain according to visual analog scale evaluation and satisfaction score.

In order to assess the clinical outcome, patients were given a four-level questionnaire (1 - perfect, 2 - good, 3 - no change, 4 - worse), indicating satisfaction scores in the $3^{\text {rd }}$ postoperative month. The outcomes of surgery was evaluated according to the following criteria: perfect (complete absence of preoperative symptoms in operated patients); good (marked reduction in preoperative symptoms but needing antibiotic therapy occasionally); no change (no reduction in preoperative symptoms, and no decrease in medical therapy requirements or in hospital admissions); and worse (increase in the exacerbations of the disease that requires hospitalization).

The visual analog scale (VAS) score was used to measure the severity of pain at post- operative $24^{\text {th }}, 48^{\text {th }}$, and $72^{\text {nd }} \mathrm{h}$. This method aims to evaluate the level of pain through a horizontal and undivided 0-10 numeral scale, named by the patient, 0 being "no pain" and 10 being "unbearable pain" [7].

All patients were informed about the surgical procedure, the risks and the complications of the procedure before the operation, and written informed consent was obtained.

\section{Surgical technique}

The VATS resection was performed using the uniportal technique. The patients were intubated with a double lumen endotracheal tube and placed in the lateral decubitus position. A utility incision measuring approximately $3-5 \mathrm{~cm}$ was performed from the 5. intercostal space in the anterior position. No rib retractor was used. A $10 \mathrm{~mm}$ 30-degree thoracoscope (Karl Storz endoscope; Karl Storz, Tuttlingen, Germany) was used to provide a panoramic appearance and it was placed in the anterior part of the

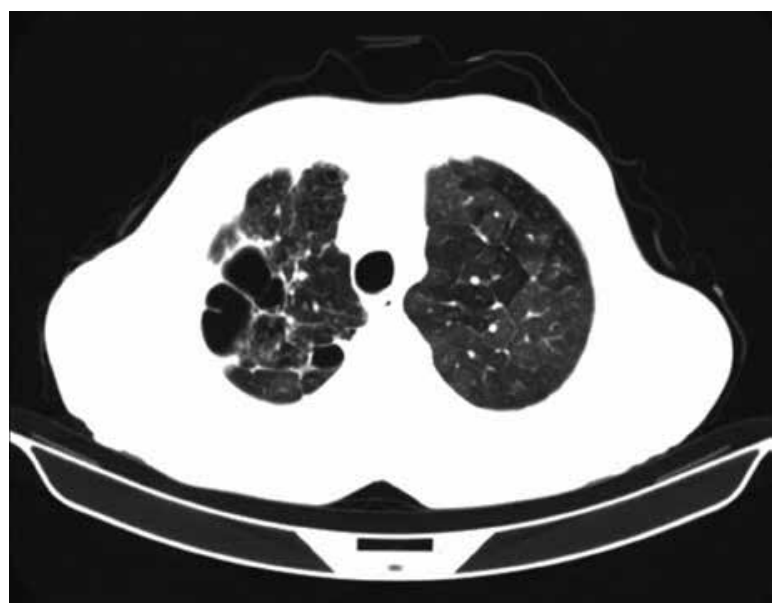

Photo 1. High resolution computed tomography (HRCT) was used for making the diagnosis of bronchiectasis 
incision. Dissecting and holding clamps used in open surgery were placed through the dorsal part of the utility incision. The surgeon was at the anterior side of the patient. First, the pleural space was evaluated for suitability of thoracoscopic anatomical resection.

The dense adhesions between the lung and thorax wall were released through the aid of a small peanut-gauze, spatula-shaped electrocautery and electro-thermal bipolar tissue sealing system (Liga-
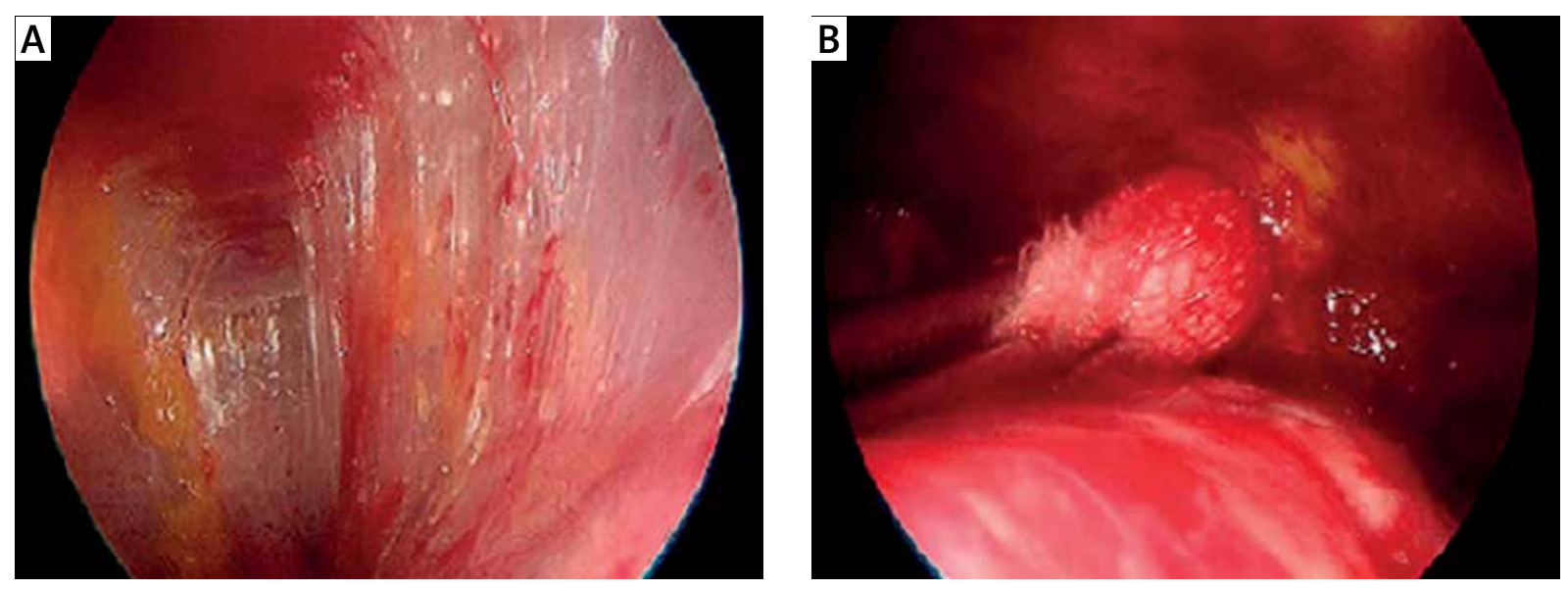

Photo 2. A-Dense pleural adhesions. B - Dissection of pleural adhesions
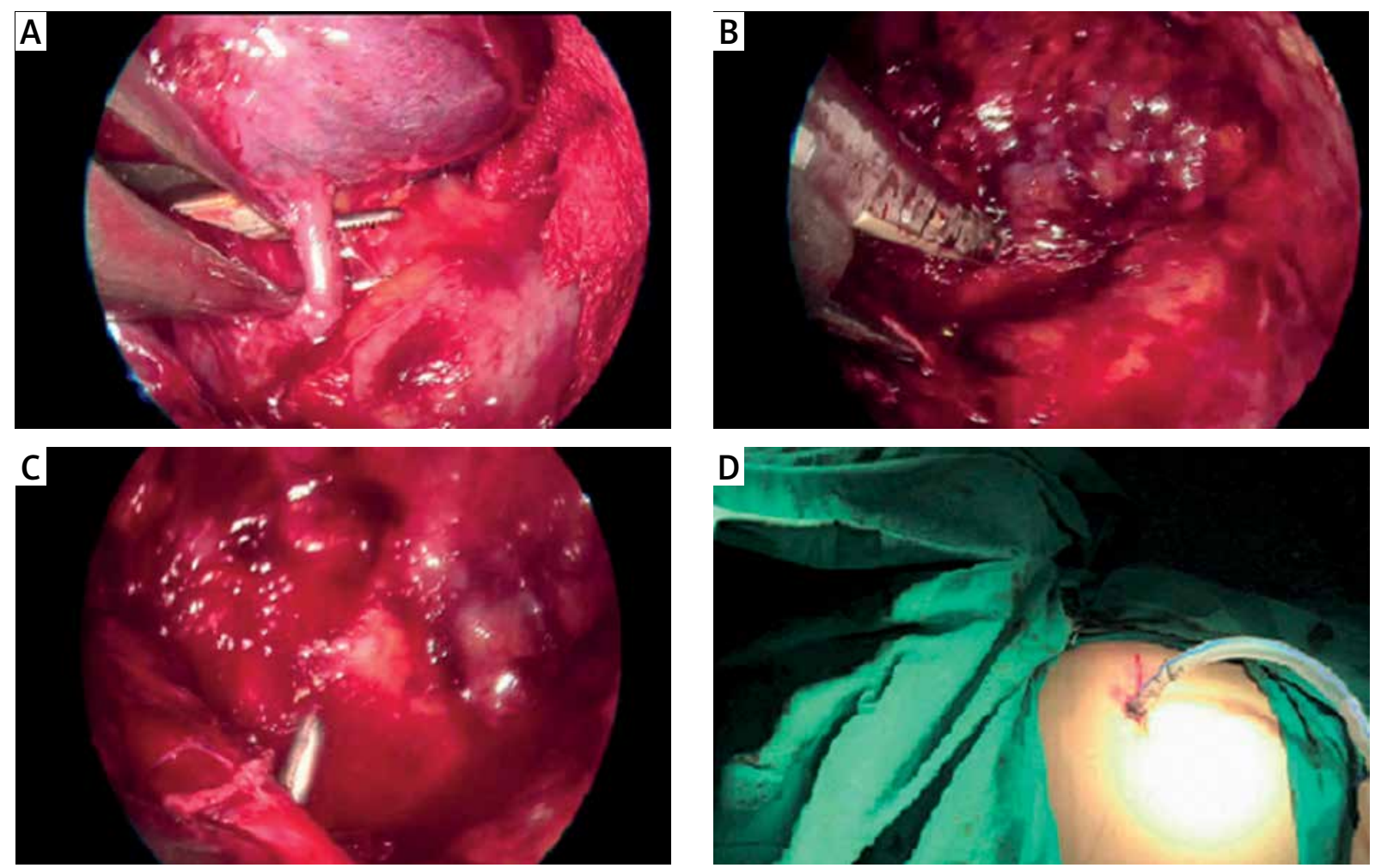

Photo 3. A - Dissection of the superior pulmonary vein. B - Division of the truncus anterior artery. C - Dissection of the upper lobe bronchi. D - A single chest tube was placed into the thorax from the same incision 


\section{Postoperative care}

All patients were given analgesia, prophylactic antibiotics and chest physiotherapy with other supportive modalities. Paracetamol was administered as the primary choice of analgesic medication in the early postoperative period. It was administered intravenously according to the severity of the pain. Tramadol hydrochloride was added only if the patient felt pain despite an adequate dose of paracetamol.

\section{Statistical analysis}

Postoperative data on clinical outcomes were collected and analyzed retrospectively and the findings were analyzed with SPSS software (SPSS Inc., Chicago, IL, USA), using the mean and standard deviation (SD) with 95\% confidence interval $(\mathrm{Cl})$. Frequency and percentage were calculated for gender, smoking status, comorbidity and presence of pleural adhesion.

\section{Results}

A total of 15 patients initially underwent single port VATS pulmonary anatomic resection and 14 procedures were completed with thoracoscopy. In one patient thoracotomy was necessary because of severe adhesions and the conversion rate was $6.6 \%$. The mean age of 14 patients was $34.5 \pm 12.1$ (range: 16-59 years) years and of the patients, 8 (57.1\%) were male and 6 (42.8\%) were female.

The presenting symptoms included productive cough in 7 (50\%) patients, excessive sputum production in $6(42.8 \%)$ patients, and hemoptysis in $4(28.5 \%)$ patients. The mean duration of symptoms was 21.3 months. There were 3 patients who had a positive microorganism that was obtained from preoperative sputum cultures in study. These patients underwent surgery after completing an adequate antibiotic regimen. A total of 3 (21.4\%) patients had comorbidities which included 1 (7.1\%) patient with asthma, 1 (7.1\%) patient with chronic obstructive pulmonary disease (COPD), and 1 (7.1\%) patient with diabetes mellitus and hypertension. One patient had a previous history of tuberculosis. The demographic data of the included patients are presented in Table I.

When we evaluate 14 patients in terms of anatomic resection, 6 patients underwent left lower lo- bectomy, 4 patients right lower lobectomy, 1 patient right upper lobectomy, 1 patient left lower lobectomy + lingulectomy, 1 patient right basal segmentectomy and 1 patient lingulectomy. The mean operative time was $137.1 \pm 24.5 \mathrm{~min}$. Pleural adhesion was observed in 5 (35.7\%) patients. An intraoperative complication developed in one patients due to hemorrhage originating from a branch of pulmonary artery during dissection, but it did not lead to open thoracotomy. The mean amount of intraoperative bleeding was $192.8 \pm 73.0 \mathrm{ml}$ and intraoperative or postoperative transfusions were not needed. The perioperative outcomes are shown in Table II.

The mean duration of chest tube was $3.57 \pm 1.65$ days and the mean drainage amount was 367.8 $\pm 151.4 \mathrm{ml}$ (range: $100-650 \mathrm{ml}$ ). The mean length of hospital stay was $4.78 \pm 1.52$ days.

The mean postoperative analgesic requirement was $2.85 \pm 0.66$ days. The VAS pain scores at postoperative $24^{\text {th }}, 48^{\text {th }}$ and $72^{\text {nd }} h$ were $3.64 \pm 0.74,3.35$ \pm 0.84 and $2.57 \pm 0.75$, respectively.

We experienced postoperative complications in two patients including atelectasis in 1 patient and prolonged air leak in the other. Atelectasis was treated with fiberoptic bronchoscopy by removing the large mucus plaque which obstructed the left upper lobe bronchus. Autologous blood pleurodesis was

Table I. Clinical characteristics of the patients

\begin{tabular}{|lc|}
\hline Characteristics & VATS $(n=14)$ \\
\hline Age [years] & $34.5 \pm 12.1$ \\
\hline Gender ratio & $1: 1$ \\
\hline \begin{tabular}{l} 
Symptom, $n(\%):$ \\
\hline Cough
\end{tabular} & $7(50)$ \\
\hline Excessive sputum production & $6(42.8)$ \\
\hline Hemoptysis & $4(28.5)$ \\
\hline Duration of symptoms [months] & 21.3 \\
\hline FEV ${ }_{1}(\%)$ predicted & 83.0 \\
\hline \begin{tabular}{l} 
Comorbidities, $n$ (\%): \\
\hline None
\end{tabular} & $11(78.5)$ \\
\hline 1 or multiple & $3(21.4)$ \\
\hline TB history, $n$ (\%) & $3(7.1)$ \\
\hline Sputum culture/positive, $n(\%)$ & $31.4)$ \\
\hline
\end{tabular}

FEV ${ }_{1}$ - forced expiratory volume in $1 \mathrm{~s}$, VATS - video-assisted thoracoscopic surgery. 
Table II. Perioperative outcomes of patients

\begin{tabular}{|lc|}
\hline Variables & VATS $(n=14)$ \\
\hline Operation time [min] & $137.1 \pm 24.5$ \\
\hline Surgical procedure, $n$ (\%): & $11(78.5)$ \\
\hline Lobectomy & $1(7.1)$ \\
\hline Lobectomy + segmentectomy & $2(14.2)$ \\
\hline Segmentectomy & $2(14.2)$ \\
\hline Pleural adhesion, $n$ (\%): & $3(21.4)$ \\
\hline Complete & $9(64.2)$ \\
\hline None & $8(57.1)$ \\
\hline Side (left), $n$ (\%) & $192.8 \pm 73.0$ \\
\hline Blood lost [ml] & $1(7.1)$ \\
\hline Perioperative complication, $n$ (\%) & $0(0)$ \\
\hline Perioperative death, $n$ (\%) & \\
\hline VATS - video-assisted thoracoscopic surgery.
\end{tabular}

chosen as the curative option for the management of prolonged air leak. There was no intraoperative or postoperative mortality.

Follow-up was completed in all patients (100\%) with a mean of 14.7 months (range: 6-30 months). Score of the patient satisfaction questionnaire in $3^{\text {rd }}$ postoperative month was $1.14 \pm 0.36$. Twelve $(85.7 \%)$ patients were "perfect" after the operation, 2 (14.2\%) "good", and $0(0 \%)$ reported "no change or worse". The postoperative outcomes are shown in Table III.

\section{Discussion}

Bronchiectasis is a disease, most often secondary to an infectious process, that results in the abnormal and permanent distortion of one or more of the conducting bronchi or airways [8]. The improvements in treatment of pulmonary infections have reduced the incidence of bronchiectasis and thereby the need for surgical treatment. However, this issue still continues to be a health problem in developing countries [9].

The goal of the surgery is to improve the quality of life of the patient and provide complete resection [10]. Surgical resection enables removal of the lung tissue composed of non-functioning bronchi, and the localized bronchiectasis fields are removed so as not to impair the structure of the neighboring tissues [11].
Table III. Postoperative outcomes of patients

\begin{tabular}{|c|c|}
\hline Variables & VATS $(n=14)$ \\
\hline Drainage volume [ml] & $367.8 \pm 151.4$ \\
\hline Chest tube removal time [days] & $3.57 \pm 1.6$ \\
\hline Length of hospital stay & $4.78 \pm 1.52$ \\
\hline Morbidity, $n$ (\%): & $2(14.2)$ \\
\hline Prolonged air leak & $1(7.1)$ \\
\hline Atelectasis & $1(7.1)$ \\
\hline Mortality, $n(\%)$ & $0(0)$ \\
\hline Follow-up period [months] & $14.7 \pm 6.8$ \\
\hline NA treatment duration [days] & $2.85 \pm 0.66$ \\
\hline \multicolumn{2}{|l|}{ Pain VAS scores: } \\
\hline $24 \mathrm{~h}$ & $3.64 \pm 0.74$ \\
\hline $48 \mathrm{~h}$ & $3.35 \pm 0.84$ \\
\hline $72 \mathrm{~h}$ & $2.57 \pm 0.75$ \\
\hline \multicolumn{2}{|c|}{ Patient satisfaction scale (3 $3^{\text {rd }}$ month), $n(\%)$ : } \\
\hline Perfect & $12(85.7)$ \\
\hline Good & $2(14.2)$ \\
\hline No change or worse & $0(0)$ \\
\hline
\end{tabular}

VATS - video-assisted thoracoscopic surgery, NA - narcotic analgesic.

The patients were evaluated with a flexible bronchoscope for the removal of secretions and for ruling out foreign bodies or endobronchial lesions. The bronchial aspirate and sputum were cultured routinely for pyogenic and tubercular bacilli. It is important to rule out bronchial obstruction secondary to a tumor or aspirated foreign body prior to attempting resection. If severe airway inflammation is found at the time of bronchoscopy, surgical therapy may be delayed until infection control is optimized.

The HRCT examination was performed to evaluate the compatibility of the patients with regards to adhesions and scar formation and also to determine the potential risks. It is the gold standard for radiologic diagnosis and location of bronchiectasis [6]. This imaging modality can detect the distribution of bronchiectatic alterations with only $2 \%$ false-negative and $1 \%$ false-positive rates [12].

Several important differences exist between anatomic resection for bronchiectasis and resection for malignancy. Typically, severe adhesions are seen in the chest wall, hilus and fissure in inflammatory dis- 
eases, and this condition is the most important factor limiting VATS resection [6]. One study reported that all benign pulmonary disease could be explored thoracoscopically; however, it was mentioned that the feasibility of resection depended on the local anatomic situation, especially adhesions [13]. Furthermore, the approach to the pulmonary arteries through the fissure may be challenging because of these adhesions and enlarged lymph nodes. The fissure may be incomplete or the bronchus and artery may be combined together.

Major complications such as bleeding may occur during the VATS procedure. This is one of the main reasons for hesitation about VATS among thoracic surgeons and thus the majority of surgeons prefer the open surgery approach in surgical treatment of bronchiectasis. However, a surgeon who has completed the learning curve can safely complete the procedure. In our study, an injury in a branch of the pulmonary artery occurred during dissection and the bleeding was controlled without requiring thoracotomy.

Compared with the right bronchus, the left bronchus has a narrower diameter, and longer mediastinal course. This is the reason for bronchiectasis being seen more commonly in the left lower lobe. The left lower lobectomy was the most common (42\%) resection localization in our study.

Since the late 1990s, VATS technique has been applied in the treatment of many other types of esophageal, mediastinal and pulmonary diseases $[4,14]$. The role of VATS has expanded substantially following the proof of causing less pain, shorter duration of hospitalization, and esthetically advantageous results $[13,15,16]$. Despite the expanding indications and advantages for VATS in thoracic surgery, reports on the use of minimally invasive techniques to accomplish surgical treatment of bronchiectasis are limited [17, 18]. Today, open surgery is widely used for anatomic resection of bronchiectasis. Unlike other thoracoscopic approaches for the treatment of bronchiectasis, this is the first study reporting the anatomic resection of bronchiectasis via the uniportal thoracoscopic approach.

Minimally invasive approaches generally soften the surgical trauma by reducing the severity of the inflammatory response. VATS allows resection via a smaller incision without separating the ribs and damaging muscle tissue. This leads to reduction of the intensity and the duration of postoperative pain, allowing patients to return to full recovery more quickly [16, 19]. Zhang et al. [18] compared multiportal VATS and thoracotomy approaches in cases with bronchiectasis in terms of the pain score and reported better results in the VATS group. In another study, Baysungur et al. reported that the postoperative pain was less in patients who underwent VATS lobectomy [17]. Corroborating the other studies, postoperative $24^{\text {th }}, 48^{\text {th }}$ and $72^{\text {nd }} \mathrm{h}$ VAS pain scores in our study were low $(3.64 \pm 0.74,3.35 \pm 0.84$ and 2.57 \pm 0.75 , respectively) and the postoperative analgesic requirement was $2.85 \pm 0.66$ days.

In addition, VATS is an advantageous method over traditional surgical techniques due to faster postoperative recovery and a shorter length of hospital stay [16, 18]. Although our study lacked a control group, it could be considered as a short hospital stay (4.78 \pm 1.52 days) in comparison to other publications about VATS.

Patients who undergo VATS have a better quality of life due to less postoperative pain and faster return to daily activity $[20,21]$. Similarly, our study found that patients had high levels of overall satisfaction in the $3^{\text {rd }}$ postoperative month (perfect $85.7 \%$, good $14.2 \%$ and no change or worse $0 \%$ ). In these patients, VATS led to significant improvement in the quality of life.

One major advantage of VATS resection is that it may be ideal for selected older patients with multiple comorbidities who would otherwise not be candidates for resection through a thoracotomy approach, as it is better tolerated owing to less chest wall injury and better preservation of postoperative lung function $[22,23]$. Brunelli et al. detected smaller changes in respiratory function values following the uniportal approach in patients who had lower cardiopulmonary reserve. They demonstrated that the uniportal approach increased the operability compared to conventional methods in high-risk patients with low respiratory function [24].

There are limited long-term outcome data for bronchiectasis patients after surgery.

In the literature, surgical treatment of bronchiectasis by a thoracotomy approach has a complication rate of between $9.4 \%$ and $24.6 \%$ and a mortality rate as high as $3.4 \%[7,25]$. In our series, the rate was $14.2 \%$, similar to rates in the literature. We had one patient with prolonged air leak and one patient with atelectasis.

This retrospective study has several limitations, such as the small sample size and the absence of a control group, making these results preliminary. 
A multicenter randomized controlled trial study with a larger sample size would be a logical next step.

\section{Conclusions}

Uniportal VATS seems to be a safe, feasible and effective technique for selected bronchiectasis patients. Fast recovery, early relief from postoperative pain, and cosmetic results are important advantages observed in this technique. Nevertheless, future prospective randomized controlled trials should be performed to confirm these preliminary results and to demonstrate the short- and long-term treatment effectiveness.

\section{Conflict of interest}

The author declares no conflict of interest.

\section{References}

1. Balkanli K, Genc O, Dakak M, et al. Surgical management of bronchiectasis: analysis and short-term results in 238 patients. Eur J Cardiothorac Surg 2003; 24: 699-702.

2. Prieto D, Bernardo J, Matos MJ, et al. Surgery for bronchiectasis. Eur I Cardiothorac Surg 2001; 20: 19-23.

3. Zu-li Z, Hui Z, Yun L, et al. Completely thoracoscopic lobectomy for the surgical management of bronchiectasis. Chin Med 2013; 126: 875-8.

4. McKenna RJ Jr, Houck W, Fuller CB. Video-assisted thoracic surgery lobectomy: experience with 1,100 cases. Ann Thorac Surg 2006; 81: 421-6.

5. Wang GS, Wang Z, Yang L, et al. Thoracoscopic management for bronchiectasis with non-tuberculous mycobacterial infection. Chin Med J 2008; 121: 2539-43.

6. Weber A, Stammberger U, Inci I, et al. Thoracoscopic lobectomy for benign disease - a single centre study on 64 cases. Eur J Cardiothorac Surg 2001; 20: 443-8.

7. Duncan JA, Bond JS, Mason T, et al. Visual analogue scale scoring and ranking: a suitable and sensitive method for assessing scar quality? Plast Reconstr Surg 2006; 118: 909-18.

8. Fishman AP, Elias JA, Fishman JA, et al. Fishman's Pulmonary Disease and Disorders. $3^{\text {rd }}$ edn. McGraw-Hill, NY 1998; 2045-70.

9. Ashour M, Al-Kattan K, Rafay MA, et al. Current surgical therapy for bronchiectasis. World J Surg 1999; 23: 1096-104.

10. Kutlay H, Cangir AK, Enön S, et al. Surgical treatment in bronchiectasis: analysis of 166 patients. Eur I Cardiothorac Surg 2002; 21: 634-7.

11. Mazières J, Murris M, Didier A, et al. Limited operation for severe multisegmental bilateral bronchiectasis. Ann Thorac Surg 2003; 75: 382-7.

12. Young K, Aspestrand F, Kolbenstvedt A. High resolution CT and bronchography in the assessment of bronchiectasis. Acta Radiol 1991; 32: 439-41.

13. Demmy TL, Nwogu C. Is video-assisted thoracic surgery lobectomy better? Quality of life considerations. Ann Thorac Surg 2008; 85: S719-28.
14. Ocakcioglu I, Alpay L, Demir M, et al. Is single port enough in minimally surgery for pneumothorax? Surg Endosc 2016; 30 : 59-64.

15. Yim AP. VATS major pulmonary resection revisited controversies, techniques, and results. Ann Thorac Surg 2002; 74: 615-23.

16. Shanthanna $H$, Aboutouk $D$, Poon E, et al. A retrospective study of open thoracotomies versus thoracoscopic surgeries for persistent postthoracotomy pain. J Clin Anesth 2016; 35: 215-20.

17. Baysungur V, Dogruyol T, Ocakcioglu I, et al. The feasibility of thoracoscopic resection in bronchiectasis. Surg Laparosc Endosc Percutan Tech 2017; 27: 194-6.

18. Zhang P, Zhang F, Jiang S, et al. Video-assisted thoracic surgery for bronchiectasis. Ann Thorac Surg 2011; 91: 239-43.

19. Bendixen M, Jorgensen OD, Kronborg, et al. Postoperative pain and quality of life after lobectomy via video-assisted thoracoscopic surgery or anterolateral thoracotomy for early stage lung cancer: a randomised controlled trial. Lancet Oncol 2016; 17: 836-44.

20. Baysungur V, Tezel C, Okur E, et al. Quality of life assessment six months after lobectomy for lung cancer: video-assisted thoracoscopic surgery versus thoracotomy. Turk Gogus Kalp Dama 2011; 19: 207-12.

21. Gazala S, Pelletier JS, Storie D, et al. A systematic review and meta-analysis to assess patient-reported outcomes after lung cancer surgery. Sci World I 2013; 2013: 789625.

22. Farjah F, Wood DE, Mulligan MS, et al. Safety and efficacy of video-assisted versus conventional lung resection for lung cancer. J Thorac Cardiovasc Surg 2009; 137: 1415-21.

23. Gonzalez-Aragoneses F, Moreno-Mata N, Simon-Adiego C, et al. Lung cancer surgery in the elderly. Crit Rev Oncol Hematol 2009; 71: 266-71.

24. Brunelli A, Xiume F, Refai M, et al. Bilateral staged uniportal VATS for synchronous lung cancers. Interact CardioVasc Thorac Surg 2006; 5: 658-9.

25. Fujimoto T, Hillejan L, Stamatis G. Current strategy for surgical management of bronchiectasis. Ann Thorac Surg 2001; 72 : $1711-5$.

Received: 24.07.2018, accepted: 3.09.2018. 